\title{
NONTRIVIAL SOLUTIONS FOR SYSTEMS OF STURM-LIOUVILLE BOUNDARY VALUE PROBLEMS
}

\author{
John R. Graef, Shapour Heidarkhani And LinguU Kong
}

Abstract. Sufficient conditions are established for the existence of at least one nontrivial classical solution to the boundary value system with Sturm-Liouville boundary conditions

$$
\left\{\begin{array}{l}
-\left(\phi_{p_{i}}\left(u_{i}^{\prime}(x)\right)\right)^{\prime}=\lambda F_{u_{i}}\left(x, u_{1}, \ldots, u_{n}\right) h_{i}\left(u_{i}^{\prime}(x)\right) \quad \text { in }(a, b), \\
\alpha_{i} u_{i}(a)-\beta_{i} u_{i}^{\prime}(a)=0, \quad \gamma_{i} u_{i}(b)+\sigma_{i} u_{i}^{\prime}(b)=0,
\end{array} \quad i=1, \ldots, n .\right.
$$

The analysis is based on variational methods and critical point theory.

Mathematics subject classification (2010): 34B15, 47J10.

Keywords and phrases: nontrivial solutions, Sturm-Liouville boundary conditions, critical point the-

ory.

\section{REFERENCES}

[1] D. Averna, G. Bonanno, Three solutions for a quasilinear two-point boundary-value problem involving the one-dimensional $p$-Laplacian, Proc. Edinburgh Math. Soc., 47 (2004), 257-270.

[2] D. Averna, G. Bonanno, A mountain pass theorem for a suitable class of functions, Rocky Mountain J. Math., 39 (2009), 707-727.

[3] A. Benmezai, J.R. Graef, L. Kong, Positive solutions to a two point singular boundary value problem, Differ. Equ. Appl., 3 (2011), 347-373.

[4] G. Bonanno, A critical point theorem via the Ekeland variational principle, Nonlinear Anal., 75 (2012), 2992-3007.

[5] G. Bonanno, P. CANDito, Non-differentiable functionals and applications to elliptic problems with discontinuous nonlinearities, J. Differential Equations, 244 (2008), 3031-3059.

[6] J.M. Davis, L. H. ERBE, J. Henderson, Multiplicity of positive solutions for higher order SturmLiouville problems, Rocky Mountain J. Math., 31 (2001), 169-184.

[7] J.R. Graef, S. Heidarkhani, L. Kong, A critical points approach for the existence of multiple solutions of a Dirichlet quasilinear system, J. Math. Anal. Appl., 388 (2012), 1268-1278.

[8] J.R. Graef, S. HeidarkHANI, L. KonG, Infinitely many solutions for systems of Sturm-Liouville boundary value problems, submitted for publication.

[9] D.D. HAI, On singular Sturm-Liouville boundary-value problems, Proc. Roy. Soc. Edinburgh Sect. A, 140 (2010), 49-63.

[10] J. Henderson, S.K. NTouyas, Positive solutions for systems of nonlinear boundary value problems, Nonlinear Stud., 15 (2008), 51-60.

[11] B. RiCCERI, A general variational principle and some of its applications, J. Comput. Appl. Math., 113 (2000), 401-410.

[12] Y. Tian, W. GE, Multiple solutions for a second-order Sturm-Liouville boundary value problem, Taiwanese J. Math., 11 (2007) 975-988.

[13] Y. Tian, W. Ge, Second-Order Sturm-Liouville boundary value problem involving the onedimensional $p$-Laplacian, Rocky Mountain J. Math., 38 (2008), 309-327.

[14] E. ZeIDler, Nonlinear Functional Analysis and its Applications, Vol. III, Springer-Verlag, BerlinHeidelberg-New York, 1985. 